\title{
Child-Computer Interaction, Ubiquitous Technologies, and Big Data
}

Juan Pablo Hourcade, University of Iowa, Alissa N. Antle, Simon Fraser University, Lisa Anthony, University of Florida, Jerry Alan Fails, Boise State University, Ole Sejer Iversen, Aarhus University, Elisa Rubegni, University of Lincoln, Mikael Skov, Aalborg University, Petr Slovak, University College London, Greg Walsh, University of Baltimore, Anja Zeising, Institut für Informationsmanagement Bremen

The children's technology landscape is changing quickly. The ubiquity of interactive technologies means children can access them just about anytime, anywhere.

At the same time, these technologies constantly collect data from and about children, bringing them into the age of big data, voluntarily or not. These developments have the potential to significantly change children's relationship to technology and the long-term impact of technology use. To discuss these changes, the child- computer-interaction community held a special interest group (SIG) meeting during the CHI 2018 conference.

Why should we care about these changes? Mobile touchscreen devices have enabled even babies to interact with digital technologies. These devices are quickly spreading throughout the world, reaching a much broader and more diverse set of demographics.

Emerging technologies such as voice agents (e.g., Echo Dot Kids Edition, a smart speaker with Alexa developed by Amazon) and neurotechnologies (e.g., KidZen, a smartphone app paired with a Neurosky EEG headset) are likely to enable more complex interactions while simultaneously collecting massive personal datasets about children.

Children are also increasingly required to use computers for schoolwork, often with online accounts hosted by multinational technology companies that collect and store, for example, all the documents they create online during their school years - even report cards (e.g., GoogleDrive, Managebac).

The result is that a wide range of children's activities now include the use of computers, and with their use comes data collection, storage, and analysis.

These forms of large-scale data collection promise the benefits of personalized experiences, potentially giving children access to technology that better fits their needs, abilities, and preferences. At the same time, there are controversial uses, such as tracking and surveillance. These uses may be beneficial to parents or schools but also could involve third parties who collect the data for unknown or unspecified uses, offering children little agency or control over their personal data during or after their childhood. For example, in most countries, there is no regulation that dictates what happens to children's data once they reach the age of majority, and often no way for them to request that it be deleted - if they are even aware that data about them exists and persists. Another data-driven domain with controversial practices is learning analytics. Modeling children's learning can bring obvious advantages in tailoring materials to their current needs and abilities. At the same time, issues may arise if the personalization is inaccurate or if it leads to stigmatization or exclusion. Additionally, children have little control or agency in the personalization process, where quantitative data is used to represent and categorize individuals with unique needs, preferences, and learning styles.

At CHI 2018, more than 40 SIG attendees considered and discussed the dimensions of ubiquity and big data as they relate to child-computer interaction. The first point was the degree to which children should have control and ownership over their data. At what ages should the level of control change? Are control, ownership, and data privacy transparent and easy to understand? The second dimension was the degree to which technologies should focus on singular or holistic outcomes. Should technologies focus on maximizing personalization and individual benefits? Or should priority be given to societal goals, such as integration and inclusion? Should technologies focus on single outcomes (e.g., learning a very specific skill) or overall benefits (e.g., learning to self- regulate)? Can both be achieved? The third dimension concerned the ways in which data may represent children superficially rather than as complex individuals with unique experiences, aspirations, and needs. When are superficial quantificationsuseful?

When can they get in the way of fully considering the complexity and depth of each child? What are the trade-offs between privacy and more accurate modeling? Other considerations included the difficulty in predicting future impacts, the temporality of each of these issues, and the fact that these technologies are not neutral, as they reflect the socioeconomic and political systems in which they are embedded and the values and goals of those who control them. 


\section{OUTCOMES OF THE SIG DISCUSSION}

Even though attendees of the SIG divided into four groups to discuss the topics outlined here, all four groups, individually and then collectively, noted the importance of education in addressing the challenges and opportunities brought by ubiquitous computing and big data for child- computer interaction. The view of education in the discussions was broad, focusing not solely on children but also on the adults involved in their lives, in the classroom and beyond. This broad view included both adults teaching children and children teaching adults (e.g., children educating their parents). Attendees identified critical issues needing to be addressed in the realm of education:

- The possible impacts of ubiquitous technologies and big data on children

- Understanding who decides what data is collected

- Understanding howalgorithms process data

- Understanding who owns and uses data and for what

- Understanding how children's data is shared (sometimes by parents) and the impacts of sharing

- Educating about issues and impacts based on age, maturity, culture, and income level

- The difficulty of predicting the consequences of technology and data use. A specific consideration brought up during discussions was that some groups of children would likely be at greater risk for data misuse than others, for example, children at risk of participating in dangerous activities or children who are more likely to struggle academically. While big data could help identify these children so they can get help, these identifications could also have negative consequences. Therefore, there is a need to carefully consider what actions to take and what data-access and persistence policies to recommend or adopt in these situations.

More broadly, outside the school context, there was a discussion of the need for ethics recommendations or guidelines directed to organizations with access to children's data, and of the possibility of regulation. However, such guidance is not straightforward to provide or implement. Many scenarios raise ethical dilemmas that require considering the trade-offs between potential benefits and negative impacts, acknowledging that some impacts may be unforeseeable. For example, smartphones that track children's locations provide the benefit of safety through surveillance, which could be countered by anxiety or a lack of control and agency that children may feel if they know their every move is being tracked. Even with clear recommendations, the largerchallenge is to persuade companies who obtain large amounts of data about children to take preventive measures to avoid its misuse. If communities and companies wait until something terrible happens, then it is likely that policymakers, who may not be as close to these issues as our community is, will step in with overly constrictive regulation, which in turn may reduce possible benefits and stifle innovation. And last, even with proactive guidelines and/or regulations, it is unclear how data misuse can be detected and how regulations would be enforced and by whom. One important matter that was raised around the issues of big data and regulation is that children must be educated to understand how data is collected, used, and stored, and how it might affect them over time.

Children's voices should be heard in the development of policies that affect them, echoing our community's longstanding commitment to children participating in the design of technologies intended to be used by them. When thinking about ethical issues and ethical control, it is important to explore these issues by learning from past research in other academic domains including bioethics, medicine, and psychology. These communities have already addressed some of these issues in non-technology-mediated situations and have more recently explored these same topics around technology developments. For example, in bioethics, medicine, and psychology, academics and practitioners have grappled with identifying an ideal age at which young people should have control over their own data, how to transition access to personal information as children become adults, and what the implications are of controlling and sharing information about children as they age. Across all the issues listed above, there is a need to consider cultural differences. Notions of individual versus collective responsibility, privacy, parenting styles, and feelings of vulnerability and trust may vary significantly across cultures. Therefore, any decisions, designs, or solutions should take into account cultural differences, provide options, and be transparent.

An obvious limitation of discussions around ethical best practices for children's interactive technologies is that it is often difficult to predict future impacts of technologies; the best we can do is include multiple voices in these discussions and look to best practices and experts (e.g., ethicists) from other domains. Given the uncertainties around future impacts, attendees recommended ongoing initiatives to create awareness about these issues (e.g., with new graduate students) and to work to continuously monitor developments in this area in order to better adapt to a changing landscape. An interdisciplinary working group focused on the ethics, benefits, and potential negative impacts related to ubiquitous technologies and big data concerning childcomputer interaction is needed.

\section{SUMMARY OF CONSIDERATIONS}

In summary, the following considerations emerged from the SIG discussion:

- Children and adults should be educated, in ways they can understand and personally relate to, about how 
ubiquitous technologies and big data may be used or misused, including acknowledging that future uses are often unknown at the time of data collection.

Children and adults should be educated about the role each can play in controlling children's data and how to weigh in on guidelines and regulations.

- Making invisible aspects of these technologies and data visible to children in ways they can understand is critical.

For example, exposing children to data and creating opportunities such that they can collect, interpret, and understand their own data would be a valuable way for them to experience, understand, and create agency about the uses of big data in their lives.

- Child-parent conversations are an important element of helping children and their parents understand the technology-mediated world they live in and will contribute to. The diversity of children, parenting styles,

family dynamics, teacher involvement, and educational practices must be considered in all recommendations.

There is a need for (diverse) societal and family involvement in the development ofany recommendations, educational initiatives, or technology developments (e.g., safety apps) that affect children.

- The tensions between safety, privacy, control, surveillance, and agency should be discussed between children and parents, in schools, and in research co-design processes.

- Consideration should be given to findings and recommendations from other academic communities across disciplines in understanding these and similar issues.

\section{MOVING FORWARD}

It was inspiring to see such thoughtful discussions, as well as the kinds of respectfully stated agreements and disagreements that characterize healthy dialogue around value-laden subjects.

While there was a general consensus that we often cannot predict future impacts or developments, there was also a shared acknowledgement that this does not negate the imperative to try and address the challenges identified in the position SIG paper [1] and the resulting SIG discussion as described above. We encourage researchers in child-computer interaction and related disciplines to consider these themes and issues as opportunities for further dialogue and debate, as well as areas for future research. 